TRANSACTIONS OF THE

AMERICAN MATHEMATICAL SOCIETY

Volume 240, June 1978

\title{
CONTINUOUS MAPS OF THE INTERVAL WITH FINITE NONWANDERING SET
}

BY

LOUIS BLOCK ${ }^{1}$

\begin{abstract}
Let $f$ be a continuous map of a closed interval into itself, and let $\Omega(f)$ denote the nonwandering set of $f$. It is shown that if $\Omega(f)$ is finite, then $\Omega(f)$ is the set of periodic points of $f$. Also, an example is given of a continuous map $g$, of a compact, connected, metrizable, one-dimensional space, for which $\Omega(g)$ consists of exactly two points, one of which is not periodic.
\end{abstract}

1. Introduction and statement of results. This paper is concerned with an analysis of the nonwandering set and periodic points (see $\$ 2$ for definitions) of continuous maps of a closed interval onto itself. Most of the paper deals with maps with finite nonwandering set.

Let $I$ be a closed interval and $f \in C^{0}(I, I)$. Let $\Omega(f)$ denote the nonwandering set of $f$, and let $P(f)$ denote the set of positive integers which occur as the period of some periodic point of $f$. Our main results are the following (see $\$ 2$ for definitions):

THEOREM A. If $\Omega(f)$ is finite, then $\Omega(f)$ is the set of periodic points of $f$.

THEOREM B. If $f$ has finitely many periodic points then for some positive integer $n, P(f)=\left\{2^{k}: k=0,1, \ldots, n\right\}$.

THEOREM C. $\Omega(f)$ is contained in the closure of the set of eventually periodic points of $f$.

EXAMPLE D. There is a continuous map $g$ of a compact, connected, metrizable, one-dimensional space, for which $\Omega(g)$ is finite, but $\Omega(g)$ is not the set of periodic points of $g$.

A major portion of this paper is devoted to proving Theorem A. We remark that since $f(\Omega(f)) \subset \Omega(f)$, it follows that if $\Omega(f)$ is finite, then for any point $x \in \Omega(f)$, the orbit of $x$ is finite. This implies that $x$ is eventually periodic (i.e. some point in the orbit of $x$ is periodic) but does not imply that $x$ is periodic. It is possible for $f \in C^{0}(I, I)$ to have points $x \in \Omega(f)$ which are eventually periodic but not periodic. In proving Theorem A, we show this cannot

Presented to the Society, January 27, 1977; received by the editors September 15, 1976.

AMS (MOS) subject classifications (1970). Primary 54H20.

'Partially supported by NSF grant MCS 76-05822. 
happen when $\Omega(f)$ is finite. The proof uses the idea of the unstable manifold which we define in $\$ 2$. Of course, the unstable manifold defined here is a modification of the unstable manifold of a hyperbolic periodic point of a differentiable map (see [5] or [6]).

The unstable manifold is a very familiar object in the context of one-to-one maps. However, other researchers have encountered difficulties in properly defining and using the unstable manifold in the context of endomorphisms (differentiable maps, not necessarily one-to-one). This is why, in $\$ 2$ of this paper, we are very careful in defining and proving elementary properties of the unstable manifold.

Theorem B is contained in a theorem of Sharkovskiy (see [7]). We include the theorem in this paper, because the proof given here is short and elementary and [7] has not been translated from the Russian.

It is true that for any positive integer $n$, there is a map $f \in C^{0}(I, I)$ with $P(f)=\left\{2^{k}: k=0, \ldots, n\right\}$. For a proof, see Lemma 16 of $[3]$.

The proof of Theorem C (given in $\$ 5$ ) is valid if the interval $I$ is replaced by the circle $S^{1}$, with the additional hypothesis that $f$ has a periodic point. This is not true of Theorems A and B.

Finally, we note that for $f \in C^{0}(I, I), \Omega(f)$ may not be the closure of the set of periodic points of $f$. See [1] for an example. Although the example is given as a mapping of the circle, it can easily be modified to a mapping of an interval.

2. Preliminary definitions and results. Let $X$ be a compact topological space, and let $f$ be a continuous map of $X$ into itself. Let $n$ be a positive integer. We define $f^{n}$ inductively by $f^{1}=f$ and $f^{n}=f \circ f^{n-1}$. Let $f^{0}$ denote the identity map.

A point $x \in X$ is said to be periodic if for some $n>0, f^{n}(x)=x$. In this case the minimum of $\left\{n>0: f^{n}(x)=x\right\}$ is called the period of $x$.

For any $x \in X$ we define the orbit of $x$ by $\operatorname{orb}(x)=\left\{f^{n}(x): n=\right.$ $0,1,2, \ldots\}$. The orbit of any periodic point will be called a periodic orbit. We say a point $x \in X$ is eventually periodic if $\operatorname{orb}(x)$ is finite (or equivalently if some element of $\operatorname{orb}(x)$ is periodic).

A point $x \in X$ is said to be wandering if for some neighborhood $V$ of $x$, $f^{n}(V) \cap V=\varnothing$ for all $n>0$. The set of points which are not wandering is called the nonwandering set and denoted by $\Omega(f) . \Omega(f)$ is a nonempty closed set and $f(\Omega(f)) \subset \Omega(f)$.

Throughout this paper we let $I$ denote a closed interval, and $C^{0}(I, I)$ denote the space of continuous maps of $I$ into itself. Let $f \in C^{0}(I, I)$ and let $p$ be a periodic point of $f$. We define the unstable manifold $W^{u}(p, f)$ as follows. Let $x \in W^{u}(p, f)$ if for any neighborhood $V$ of $x, x \in f^{n}(V)$ for some positive integer $n$. If $p$ is a fixed point of $f$ we define $W^{u}(p, f,+)$ and 
$W^{u}(p, f,-)$ as follows. Let $x \in W^{u}(p, f,+)$ if for every interval $K$ with left endpoint $p, x \in f^{n}(K)$ for some positive integer $n$. Let $x \in W^{u}(p, f,-)$ if for every interval $K$ with right endpoint $p, x \in f^{n}(K)$ for some positive integer $n$.

We will now prove some basic results concerning $W^{u}(p, f)$. It will be helpful for the reader, in following most of the proofs in this paper, to draw an interval and label points in the correct order.

Lemma 1. Let $f \in C^{0}(I, I)$. If $p$ is a fixed point of $f$, then $W^{u}(p, f)$ is connected.

Proof. Let $b$ and $c$ be points in $W^{u}(p, f)$ and suppose $b<x<c$. Without loss of generality we may assume that $x \geqslant p$. Let $V$ be any open interval about $p$. Then for some $n>0, c \in f^{n}(V)$. Since $f^{n}(V)$ is an interval containing $p$ and $c$, we have $x \in f^{n}(V)$. Hence $x \in W^{u}(p, f)$. Q.E.D.

LeMMA 2. Let $f \in C^{0}(I, I)$ and let $\left\{p_{1}, \ldots, p_{n}\right\}$ be a periodic orbit of $f$. Then

$$
W^{u}\left(p_{1}, f\right)=W^{u}\left(p_{1}, f^{n}\right) \cup \cdots \cup W^{u}\left(p_{n}, f^{n}\right) .
$$

Proof. By renumbering we may assume that $f\left(p_{i}\right)=p_{i+1}$ for $i=1, \ldots, n$ -1 and $f\left(p_{n}\right)=p_{1}$. First we show that

$$
W^{u}\left(p_{1}, f\right) \subset W^{u}\left(p_{1}, f^{n}\right) \cup \cdots \cup W^{u}\left(p_{n}, f^{n}\right) .
$$

Suppose $z \in W^{u}\left(p_{1}, f\right)$ and

$$
z \notin W^{u}\left(p_{1}, f^{n}\right) \cup \cdots \cup W^{u}\left(p_{n}, f^{n}\right) .
$$

For each $i=1, \ldots, n$, there is a neighborhood $V_{i}$ of $p_{i}$ such that $z \notin \cup_{m=0}^{\infty}$ $f^{n m}\left(V_{i}\right)$. Let $W_{1}=V_{1}$ and for each $j=2, \ldots, n$ let $W_{j}$ be a neighborhood of $p_{1}$, with $f^{j-1}\left(W_{j}\right) \subset V_{j}$. Let $W_{0}=W_{1} \cap \cdots \cap W_{n}$. Then $z \notin \cup_{m=0}^{\infty}$ $f^{m}\left(W_{0}\right)$. This contradicts $z \in W^{u}\left(p_{1}, f\right)$. Hence

$$
W^{u}\left(p_{1}, f\right) \subset W^{u}\left(p_{1}, f^{n}\right) \cup \cdots \cup W^{u}\left(p_{n}, f^{n}\right) .
$$

We now show that

$$
W^{u}\left(p_{1}, f^{n}\right) \cup \cdots \cup W^{u}\left(p_{n}, f^{n}\right) \subset W^{u}\left(p_{1}, f\right) .
$$

Let $z \in W^{u}\left(p_{k}, f^{n}\right)$ for some $k=1, \ldots, n$. Let $V$ be any neighborhood of $p_{1}$. If $k=1$, let $N=V$. If $k>1$, let $N$ be a neighborhood of $p_{k}$ with $f^{n-k+1}(N) \subset V$. Since $z \in W^{u}\left(p_{k}, f^{n}\right), z \in f^{n m}(N)$ for some $m>0$. Hence $z \in f^{r}(V)$ where $r=n m$ if $k=1$, and $r=n m-(n-k+1)$ if $k>1$. Thus $z \in W^{u}\left(p_{1}, f\right)$. Q.E.D.

Lemma 3. Let $f \in C^{0}(I, I)$ and let $p$ be a periodic point of $f$. Let $J=$ $W^{u}(p, f)$. Then $f(J)=J$.

Proof. First we show $f(J) \subset J$. Let $x \in J$. Then for any neighborhood $W$ 
of $p, x \in f^{m}(W)$ for some positive integer $m$. Hence $f(x) \in f^{m+1}(W)$. Thus $f(x) \in J$ and $f(J) \subset J$.

We now show that $f$ maps $J$ onto $J$. Suppose $f(J)$ is a proper subset of $J$. Let $z \in J-f(J)$, and let $n$ be the period of $p$. By Lemma 2, $z \in W^{u}\left(p_{0}, f^{n}\right)$ for some $p_{0} \in \operatorname{orb}(p)$. Let $K=W^{u}\left(p_{0}, f^{n}\right)$.

First suppose that $K$ is a neighborhood of $p_{0}$. Then $z \in f^{n m}(K)$ for some positive integer $m$. Note that since $f(J) \subset J, f^{r}(J) \subset J$ for every positive integer $r$. Hence

$$
f^{n m}(K) \subset f^{n m}(J) \subset f\left(f^{n m-1}(J)\right) \subset f(J) .
$$

Thus $z \in f(J)$, a contradiction.

Now suppose that $K$ is not a neighborhood of $p_{0}$. Then $K$ must be an interval with one endpoint $p_{0}$. Without loss of generality we may assume $K=\left[p_{0}, b\right]$ for some $b \in I$.

Choose $c<p_{0}$ such that $\forall x \in\left[c, p_{0}\right], f^{n}(x) \neq z$. This can be done by continuity of $f^{n}$, since $f^{n}\left(p_{0}\right)=p_{0}$. Since $c<p_{0}, c \notin K$. Hence, there is a neighborhood $V=(a, d)$ of $p_{0}$, with $c<a<p_{0}<d<z$, such that $c \notin$ $\cup_{m=0}^{\infty} f^{n m}(V)$. Note that for any positive integer $m, z \notin f^{n m}(K)$ because $f^{n m}(K) \subset f(J)$. Now $c \notin f^{n}(V)$ by choice of $V$. Also $z \notin f^{n}(V)$. This is true since $V=\left(a, p_{0}\right) \cup\left[p_{0}, d\right)$, and $z \notin f^{n}\left(a, p_{0}\right)$ by choice of $c$, while $z \notin$ $f^{n}\left(\left[p_{0}, d\right)\right)$ because $\left[p_{0}, d\right) \subset K$. Since $f^{n}(V)$ is an interval containing $p_{0}$, $f^{n}(V) \subset(c, z)$. By repeating the above argument inductively, it follows that for any positive integer $m, z \notin f^{n m}(V)$. This is a contradiction, since $z \in K$ and $K=W^{u}\left(p_{0}, f^{n}\right)$. Q.E.D.

Lemma 4. Let $f \in C^{0}(I, I)$ and let $p$ be a periodic point of $f$. Let $J=$ $W^{u}(p, f)$, and let $\bar{J}$ denote the closure of $J$. Then any element of $\bar{J}-J$ is periodic.

Proof. Let $x \in \bar{J}-J$. By Lemma $3, f(\bar{J})=\bar{J}$, so $x$ must have an inverse image $y \in \bar{J}$. Since $f(J)=J, y \notin J$. Hence $y \in \bar{J}-J$. Thus $f(\bar{J}-J) \supset \bar{J}$ $-J$.

It follows from Lemmas 1 and 2 that $\bar{J}-J$ is a finite set. Hence $f$ maps $\bar{J}-J$ homeomorphically onto itself. This implies that every point in $\bar{J}-J$ is periodic. Q.E.D.

LeMma 5. Let $f \in C^{0}(I, I)$. Let $K \subset I$ be a closed interval with $K \subset f(K)$. Then $f$ has a fixed point in $K$.

Proof. For some points $x$ and $y$ in $K, f(x)$ is the left endpoint of $f(K)$, and $f(y)$ is the right endpoint of $f(K)$. Hence $f(x)<x$ and $f(y)>y$. By continuity, for some $z$ in the closed interval joining $x$ and $y, f(z)=z$. Q.E.D. 


\section{Proof of Theorem A.}

LeMMa 6. Let $f \in C^{0}(I, I)$. Suppose $f$ has finitely many periodic points, and $p$ is a fixed point of $f$. Let $x \in W^{u}(p, f)$. If $x>p$, then $x \in W^{u}(p, f,+)$. If $x<p$, then $x \in W^{u}(p, f,-)$.

Proof. Since the two assertions are analogous we will prove only the first. Let $x \in W^{u}(p, f)$ and $x>p$.

Suppose $x \notin W^{u}(p, f,+)$. Then $x \in W^{u}(p, f,-)$. Let $p_{1}$ be the closest fixed point to $p$ which is less than $p$ (or let $p_{1}$ be the left endpoint of $I$ if there are no fixed points less than $p$ ). Then $\forall y \in\left(p_{1}, p\right), f(y)<y$ (because if $f(y)>y \quad \forall y \in\left(p_{1}, p\right)$, then $W^{u}(p, f,-) \subset W^{u}(p, f,+)$ and $x \in$ $W^{u}(p, f,+)$ a contradiction).

Let $z$ be the supremum of $\left\{y<p_{1}: f(y)=p\right\}$. This set is nonempty since $x \in W^{u}(p, f,-)$. Note that $f(z)=p$ and $z<p_{1}$.

Let $y$ be any point with $z<y<p_{1}$. Then $f([z, y])$ is an interval $[f(y), p]$. Since $x \in W^{u}(p, f,-)$, it follows that $z \in W^{u}(p, f,-)$. Hence $z \in$ $f^{n}([f(y), p])$ for some $n>0$. This implies that $z \in f^{n+1}([z, y])$. Since $f^{n+1}([z, y])$ is an interval containing $z$ and $p, f^{n+1}([z, y]) \supset[z, y]$. By Lemma $5, f$ has a periodic point in $[z, y]$. Since $y$ was arbitrary, $f$ has infinitely many periodic points, a contradiction. Q.E.D.

THEOREM 7. Let $f \in C^{0}(I, I)$. Suppose $f$ has finitely many periodic points, and $p$ is a fixed point of $f$. If $x \in W^{u}(p, f)$ and $f(x)=p$, then $x=p$.

Proof. Suppose $x \in W^{u}(p, f)$ with $f(x)=p$, and $x \neq p$. Without loss of generality we may assume that $x>p$. By Lemma $5, x \in W^{u}(p, f,+)$. This implies that $f([p, x]) \neq\{p\}$. Hence for some interval $(q, z) \subset(p, x), f^{-1}(p)$ $\cap(q, z)=\varnothing$ and $f(z)=p$. Thus for any $a \in I$ with $a<z, f([a, z \rrbracket)$ is an interval containing $p$.

Suppose the following is true:

(1) For any $a \in I$ with $a<z, f([a, z])$ contains an interval of the form $[p, b]$.

Let $a \in I$ with $p<a<z$. Then $f([a, z]) \supset[p, b]$ for some $b \in I$. Since $z \in W^{u}(p, f,+)$, for some $n>0, z \in f^{n}([p, b])$. Hence $z \in f^{n+1}([a, z])$. Now $f^{n+1}([a, z])$ is an interval containing $p$ and $z$. Hence $f^{n+1}([a, z]) \supset[a, z]$. By Lemma $5, f$ has a periodic point in $[a, z]$. Since $a$ was an arbitrary point (with $p<a<z) f$ has infinitely many periodic points, a contradiction. Hence (1) is not true.

Thus the following must be true:

(2) For any $a \in I$ with $a<z, f([a, z])$ contains an interval of the form $[b, p]$.

We claim that for some $y \in(p, z), f(y)>p$. To prove this, suppose for all 
$y \in(p, z), f(y)<p$. Then $z \in W^{u}(p, f,-)$. Thus for any $a \in I$, with $p<a<z, f^{n}([a, z]) \supset[a, z]$ for some $n>0$. Hence $f$ has infinitely many periodic points, a contradiction. This establishes the claim that for some $y_{0} \in(p, z), f\left(y_{0}\right)>p$.

Let $d$ be the infimum of $\left\{v>y_{0}: f(v)=p\right\}$. Then $f(d)=p$, and $y_{0}<d<$ z. Let $a \in I$ with $p<a<d$. Then $f([a, d])$ contains an interval of the form $[p, b]$ (for some $b \in I)$. Since $d \in W^{u}(p, f,+)$ (as $W^{u}(p, f,+)$ is an interval containing $p$ and $x$ ), for some $n>0, f^{n}([a, d]) \supset[a, d]$. Since $a$ was an arbitrary point with $p<a<d, f$ has infinitely many periodic points. This is a contradiction. Q.E.D.

THEOREM 8. Let $f \in C^{0}(I, I)$ and suppose $f$ has finitely many periodic points. Let $\left\{p_{1}, \ldots, p_{n}\right\}$ be a periodic orbit of $f$ (of period $\left.n\right)$. If $p_{i}$ and $p_{j}$ are distinct elements of $\left\{p_{1}, \ldots, p_{n}\right\}$ then $p_{j} \notin W^{u}\left(p_{i}, f^{n}\right)$.

Proof. Suppose $p_{i}$ and $p_{j}$ are distinct elements of $\left\{p_{1}, \ldots, p_{n}\right\}$ with $p_{j} \in W^{u}\left(p_{i}, f^{n}\right)$. We claim that for each $k=1, \ldots, n, W^{u}\left(p_{k}, f^{n}\right)$ contains an element of $\left\{p_{1}, \ldots, p_{n}\right\}-\left\{p_{k}\right\}$. To prove this, let $V$ be any neighborhood of $p_{k}$. Let $r$ be the smallest positive integer with $f^{r}\left(p_{i}\right)=p_{k}$. There is a neighborhood $W$ of $p_{i}$ with $f^{r}(W) \subset V$. Now for some $m>0, p_{j} \in f^{n m}(W)$. Hence

$$
f^{r}\left(p_{j}\right) \in f^{r}\left(f^{n m}(W)\right)=f^{n m}\left(f^{r}(W)\right) \subset f^{n m}(V) .
$$

Since $V$ was arbitrary, $f^{r}\left(p_{j}\right) \in W^{u}\left(p_{k}, f^{n}\right)$. Also, $f^{r}\left(p_{i}\right)=p_{k}$ and $p_{i} \neq p_{j}$ imply that $f^{r}\left(p_{j}\right) \neq p_{k}$. This proves the claim.

By renumbering, we may assume that $p_{1}<p_{2}<\cdots<p_{n}$. Since $W^{u}\left(p_{1}, f^{n}\right)$ is an interval containing $p_{1}$ and some element of $\left\{p_{1}, \ldots, p_{n}\right\}-$ $\left\{p_{1}\right\}, p_{2} \in W^{u}\left(p_{1}, f^{n}\right)$. Similarly, either $p_{1} \in W^{u}\left(p_{2}, f^{n}\right)$ or $p_{3} \in W^{u}\left(p_{2}, f^{n}\right)$.

Suppose $p_{1} \in W^{u}\left(p_{2}, f^{n}\right)$. By Lemma $6, p_{2} \in W^{u}\left(p_{1}, f^{n},+\right)$ and $p_{1} \in$ $W^{u}\left(p_{2}, f^{n},-\right)$. Since $\left[p_{1}, p_{2}\right] \subset W^{u}\left(p_{1}, f^{n}\right)$, it follows from Theorem 7 , that for all $x \in\left(p_{1}, p_{2}\right), f^{n}(x)>p_{1}$. So $p_{2} \in W^{u}\left(p_{1}, f^{n},+\right)$ implies that for some $x \in\left(p_{1}, p_{2}\right), f^{n}(x)=p_{2}$. Let

$$
z=\inf \left\{x \in\left(p_{1}, p_{2}\right): f^{n}(x)=p_{2}\right\} .
$$

Then $z \in\left(p_{1}, p_{2}\right)$ and $f^{n}(z)=p_{2}$. Let $p_{1}<a<z$. Then $f^{n}([a, z])$ contains an interval of the form $\left[b, p_{2}\right]$. Since $p_{1} \in W^{u}\left(p_{2}, f^{n},-\right)$, for some $m>0$, $f^{n m}([a, z]) \supset[a, z]$. By Lemma $5, f$ has a periodic point in $[a, z]$. Since $a$ was an arbitrary point with $p_{1}<a<z, f$ has infinitely many periodic points. This is a contradiction, and so $p_{1} \notin W^{u}\left(p_{2}, f^{n}\right)$. Hence $p_{3} \in W^{u}\left(p_{2}, f^{n}\right)$.

By the same argument, it follows that $p_{i+1} \in W^{u}\left(p_{i}, f^{n}\right)$ for $i=1, \ldots, n$ - 1. In particular, $p_{n} \in W^{u}\left(p_{n-1}, f^{n}\right)$. But since $W^{u}\left(p_{n}, f^{n}\right)$ is an interval containing $p_{n}$ and some element of $\left\{p_{1}, \ldots, p_{n-1}\right\}, p_{n-1} \in W^{u}\left(p_{n}, f^{n}\right)$. This implies (by the same argument as the preceding paragraph) that $f$ has 
infinitely many periodic points, a contradiction. Q.E.D.

THEOREM 9. Let $f \in C^{0}(I, I)$ and suppose $\Omega(f)$ is finite. Let $x \in \Omega(f)$ and suppose $x$ is not periodic. Then for some periodic point $p$ of $f, \exists z \in W^{u}(p, f)$ such that $f(z)=p$ and $z$ is not periodic.

Proof. $x \in \Omega(f)$ implies that $f^{m}(x) \in \Omega(f), \forall m>0$. Since $\Omega(f)$ is finite, this implies that $x$ is eventually periodic. Hence $\exists z \in \operatorname{orb}(x)$ such that $f(z)=p$ for some periodic point $p$, but $z$ is not periodic. Since $z \in \operatorname{orb}(x)$, $z \in \Omega(f)$. By Lemma 4, to prove the theorem, it suffices to show that $z \in \overline{W^{u}(p, f)}$. Suppose $z \notin \overline{W^{u}(p, f)}$.

Let $(a, b)$ be an open interval containing $z$, with $[a, b] \cap W^{u}(p, f)=\varnothing$. Since $a \notin W^{u}(p, f)$ and $b \notin W^{u}(p, f)$, there is an open interval $N$ containing $p$, such that $f^{m}(N) \cap\{a, b\}=\varnothing$ for every positive integer $m$. Now, for each positive integer $m, f^{m}(N)$ is an interval which contains some element of $\operatorname{orb}(p)$. Since $\operatorname{orb}(p) \subset W^{u}(p, f), \operatorname{orb}(p) \cap(a, b)=\varnothing$. Hence $f^{m}(N) \cap(a, b)=\varnothing$ for every pòsitive integer $m$.

By choosing $N$ smaller if necessary, we may assume that $N \cap(a, b)=\varnothing$. Let $V$ be a neighborhood of $z$ with $V \subset(a, b)$ and $f(V) \subset N$. Then $f^{m}(V) \cap$ $V=\varnothing$ for every positive integer $m$. This is a contradiction since $z \in \Omega(f)$. Q.E.D.

TheOREM A. Let $f \in C^{0}(I, I)$ and suppose $\Omega(f)$ is finite. Then $\Omega(f)$ is the set of periodic points of $f$.

Proof. Suppose $x \in \Omega(f)$ and $x$ is not periodic. By Theorem 9, for some periodic point $p_{1}, \exists z \in W^{u}\left(p_{1}, f\right)$ such that $f(z)=p_{1}$ and $z$ is not periodic. Let $n$ be the period of $p_{1}$ and let $\operatorname{orb}\left(p_{1}\right)=\left\{p_{1}, \ldots, p_{n}\right\}$. By Lemma 2, $z \in W^{u}\left(p_{k}, f^{n}\right)$ for some $p_{k} \in\left\{p_{1}, \ldots, p_{n}\right\}$.

Note that $f^{n}(z) \in\left\{p_{1}, \ldots, p_{n}\right\}$ and (by Lemma 3) $f^{n}(z) \in W^{u}\left(p_{k}, f^{n}\right)$. Hence, by Theorem $8, f^{n}(z)=p_{k}$. Since $\Omega(f)$ is finite, $f^{n}$ has only finitely many periodic points. Also, $z \in W^{u}\left(p_{k}, f^{n}\right)$ and $f^{n}(z)=p_{k}$. This implies, by Theorem 7, that $z=p_{k}$. This is a contradiction, because $z$ is not periodic. Q.E.D.

\section{Proof of Theorem B.}

THeOREM 10. Let $f \in C^{0}(I, I)$, suppose $f$ has a periodic point which is not fixed. Then $f$ has a periodic point of period 2 .

Proof. We may assume $f$ has a periodic point of period greater than 2, or else the theorem is proved. Let $n$ be the smallest element of $\{m>3: f$ has a periodic point of period $m\}$. Let $\left\{x_{1}, \ldots, x_{n}\right\}$ be a periodic orbit of period $n$, with $x_{i}<x_{i+1}$ for $i=1, \ldots, n-1$.

Let $I_{k}=\left[x_{k}, x_{k+1}\right]$ for $k=1, \ldots, n-1$. Note that for each $I_{k}, f\left(I_{k}\right) \supset I_{j}$ for some $j \neq k$. Hence for some set of distinct $I_{k}$ 's, $\left\{I_{k_{1}}, \ldots, I_{k_{m}}\right\}, f\left(I_{k_{1}}\right) \supset$ 
$I_{k_{1+1}}$ for $i=1, \ldots, m-1$ and $f\left(I_{k_{m}}\right) \supset I_{k_{1}}$, where $2<m<n-1$.

Let $J_{k_{m}}$ be a closed interval with $J_{k_{m}} \subset I_{k_{m}}$ and $f\left(J_{k_{m}}\right)=I_{k_{1}}$. Also, for $i=1, \ldots, m-1$ let $J_{k_{1}}$ be a closed interval with $J_{k_{i}} \subset I_{k_{i}}$ and $f\left(J_{k_{i}}\right)=J_{k_{i+1}}$. Then $f^{m}\left(J_{k_{1}}\right)=I_{k_{1}}$. By Lemma 5, $f^{m}$ has a fixed point $y \in J_{k_{1}}$. Since $m<n$, $\operatorname{orb}(y) \cap\left\{x_{1}, \ldots, x_{n}\right\}=\varnothing$. Hence $f^{i}(y)$ is in the interior of $I_{k_{i+1}}$ for $i=$ $1, \ldots, m-1$. Since the $i_{k}$ 's have pairwise disjoint interiors, $y$ is a periodic point of $f$ of period $m$. It follows from the choice of $n$, and the fact that $m<n$, that $m=2$. Q.E.D.

THEOREM 11. Let $f \in C^{0}(I, I)$. Suppose $f$ has a periodic point whose period is not a power of 2. Then for each positive integer $k, f$ has a periodic point of period $2^{k}$. (In particular, $f$ has infinitely many periodic points.)

Proof. Let $k$ be a positive integer and $n=2^{(k-1)}$. Then $f^{n}$ has a periodic point which is not fixed. By Theorem $10, f^{n}$ has a periodic point $x$ of period 2 . Since $n=2^{(k-1)}, x$ is a periodic point of $f$ of period $2^{k}$. Q.E.D.

THEOREM B. Let $f \in C^{0}(I, I)$ and suppose $f$ has finitely many periodic points. Let $P(f)$ denote the set of positive integers which occur as the period of some periodic point of $f$. Then for some nonnegative integer $n, P(f)=\left\{2^{k}: k=\right.$ $0,1, \ldots, n\}$.

Proof. By Theorem 11, there is a nonnegative integer $n$, such that $P(f) \subset$ $\left\{2^{k}: k=0,1, \ldots, n\right\}$, and $2^{n} \in P(f)$. If $n \in\{0,1\}$, the theorem follows immediately, so we may assume $n>2$.

Let $j=2^{(n-2)}$. Then $f^{j}$ has a periodic point of period 4. By Theorem $10, f^{j}$ has a periodic point of period 2. Hence $f$ has a periodic point of period $2^{n-1}$.

Repeating the above argument, it follows that $P(f)=\left\{2^{k}: k=\right.$ $0,1, \ldots, n\}$. Q.E.D.

\section{Proof of Theorem $C$.}

THEOREM C. Let $f \in C^{0}(I, I)$. Then $\Omega(f)$ is contained in the closure of the set of eventually periodic points of $f$.

Proof. Suppose the statement is false. Let $V$ be the complement in $I$ of the closure of the set of eventually periodic points of $f$. Then $V \cap \Omega(f) \neq \varnothing$.

Let $x \in V \cap \Omega(f)$. Let $W$ be the component of $V$ with $x \in W$. Since $V$ is open in $I, W$ is an interval and $W$ is a neighborhood of $x$.

Let $n$ be the smallest element of $\left\{m>0: f^{m}(W) \cap W \neq \varnothing\right\}$. This set is nonempty since $x \in \Omega(f)$. Since $f^{n}(W) \cap W \neq \varnothing$, and no point of $W$ is eventually periodic, it follows that $f^{n}(W) \subset \bar{W}$. This fact and the choice of $n$, imply that $x \in \Omega\left(f^{n}\right)$.

Since there are no periodic points in $W$, either $\forall y \in W, f^{n}(y)>y$ or 
$\forall y \in W, f^{n}(y)<y$. Without loss of generality, we may assume that $\forall y \in$ $W, f^{n}(y)>y$.

Let $K$ be a closed interval which contains a neighborhood of $x$ (in $I$ ) with $K \subset W$. Let $d$ be the minimum value of the function $g$ defined on $K$ by $g(y)=f^{n}(y)-y$. Then $d>0$.

Let $N$ be an interval of length smaller than $d$ and a neighborhood of $x$, with $N \subset K$. Then $f^{n}(N) \cap N=\varnothing$. Since $f^{n}(y)>y, \forall y \in W, f^{n m}(N) \cap N$ $=\varnothing, \forall m>0$. This is a contradiction, since $x \in \Omega\left(f^{n}\right)$. Q.E.D.

\section{Example D.}

EXAMPLE D. There is a continuous map $g$ of a compact, connected, metrizable, one-dimensional space $X$, for which $\Omega(g)$ is finite, but $\Omega(g)$ is not the set of periodic points of $g$.

Proof. Let $S$ be any circle in the plane, and let $A$ be any arc in the plane joining two distinct points on $S$ such that $S \cap A$ consists of exactly two points. Let $X=S \cup A$ (see Figure 1). Then $X$ is a compact, connected, metrizable, one-dimensional space (with the topology induced by the usual topology on the plane).

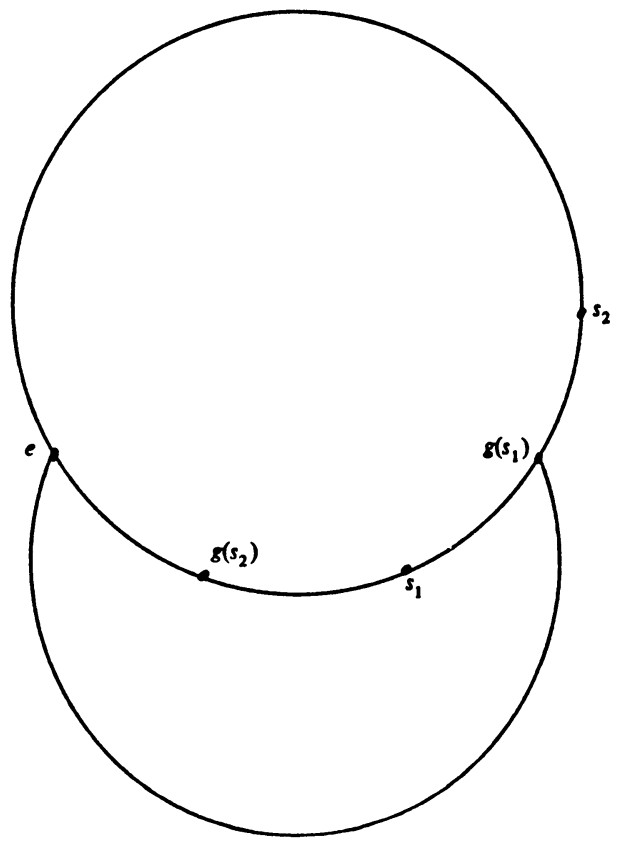

FIGURE 1

Let $(a, b)$ (respectively $[a, b])$ denote the open (respectively closed) arc on $S$ from $a$ counterclockwise to $b$. Let $g$ be a continuous map of $X$ into itself, as 
pictured in Figure 1, with the following properties.

(1) $g$ has exactly one fixed point, $e$.

(2) $S \cap A=\left\{e, g\left(s_{1}\right)\right\}$.

(3) $g$ maps the interval $\left[e, s_{1}\right]$ homeomorphically onto $\left[e, g\left(s_{1}\right)\right]$.

(4) $g$ maps the interval $\left[s_{1}, g\left(s_{1}\right)\right]$ homeomorphically onto the $\operatorname{arc} A$, with $g\left(g\left(s_{1}\right)\right)=e$.

(5) $g$ maps the interval $\left[g\left(s_{1}\right), s_{2}\right]$ homeomorphically onto $\left[e, g\left(s_{2}\right)\right]$.

(6) $g$ maps the interval $\left[s_{2}, e\right]$ homeomorphically onto $\left[e, g\left(s_{2}\right)\right]$.

(7) $g(x)=e, \forall x \in A$.

We will show that $\Omega(g)=\left\{e, g\left(s_{1}\right)\right\}$. All points in $\left(g\left(s_{1}\right), e\right)$ are wandering, because these points are not in the image of $g$. Also, all points in $A-$ $\left\{e, g\left(s_{1}\right)\right\}$ are wandering by Property (7) above.

For any $x \in\left(e, g\left(s_{1}\right)\right)$ there is a neighborhood $V$ of $x$ and a positive integer $n$ with $g^{n}(V)=\{e\}$. Thus, all points in $\left(e, g\left(s_{1}\right)\right)$ are wandering. However, $g\left(s_{1}\right)$ is nonwandering. To see this let $V$ be a neighborhood of $g\left(s_{1}\right)$. Then $g(V)$ contains an interval on $S$ of the form $[e, b]$. Hence $g^{n}(V) \cap V \neq \varnothing$, for some $n>0$.

Thus $\Omega(g)=\left\{e, g\left(s_{1}\right)\right\}$. So $\Omega(g)$ is finite, but $\Omega(g)$ contains a point which is not periodic. Q.E.D.

\section{REFERENCES}

1. L. Block, Diffeomorphisms obtained from endomorphisms, Trans. Amer. Math. Soc. 214 (1975), 403-413.

2. Morse-Smale endomorphisms of the circle, Proc. Amer. Math. Soc. 48 (1975), 457-463.

3. The periodic points of Morse-Smale endomorphisms of the circle, Trans. Amer. Math. Soc. 226 (1977), 77-88.

4. R. Bowen and J. Franks, The periodic points of maps of the disk and the interval, Topology 15 (1976), 337-442.

5. M. Shub, Endomorphisms of compact differentiable manifolds, Amer. J. Math. 91 (1969), 175-199. MR 39 \#2169.

6. S. Smale, Differentiable dynamical systems, Bull. Amer. Math. Soc. 73 (1967), 747-817. MR 37 \#3598.

7. A. N. Sarkovskii, Co-existence of cycles of a continuous mapping of the line into itself, Ukrain. Math. Z.16 (1964), 61-71. (Russian) MR 28 \#3121.

Department of Mathematics, University of Florida, Gainesville, Florida 32611 